\title{
Low-field helicon discharges
}

\author{
F F Chen $\dagger \ddagger$, X Jiang $\dagger$, J D Evans $\dagger$, G Tynan $\ddagger$ and D Arnush $\dagger$ \\ $\dagger$ University of California, Los Angeles, CA 90095-1594, USA \\ $\ddagger$ PMT, Inc., Chatsworth, CA 91311, USA
}

\begin{abstract}
Operation of helicon discharges at magnetic fields $B_{0}$ below $100 \mathrm{G}$ is of interest for plasma etching and deposition reactors if high ion flux can be maintained with reduced field requirements. The theory of coupled helicon and Trivelpiece-Gould modes is summarized for uniform $B_{0}$. Initial results from two experiments are reported. The first has a single $5 \mathrm{~cm}$ diameter tube with $B_{0}=0-100 \mathrm{G}$ injecting plasma into a field-free region. The second contains a two-dimensional array of seven such tubes covering a large area. Densities and density profiles are measured for various fields, RF powers and gas pressures. The highest density generally occurs at zero field. Because of the non-uniformity in $B_{0}$, direct comparison with theory cannot yet be made.
\end{abstract}

\section{Introduction}

Experiments on three different helicon machines, now dismantled, showed previously that a density peak occurs for magnetic fields between 10 and $50 \mathrm{G}$. This was attributed to the occurrence of an electron cyclotron wave, or Trivelpiece-Gould (TG) mode, when $\omega / \omega_{\mathrm{c}}$ was of order unity. This effect was not easily reproducible, however, and the purpose of the present work is to study systematically the coupling of helicon waves to TG waves, and the transition to ICP (inductively coupled plasma) operation at zero field, both theoretically and experimentally for the purpose of developing economic, uniform plasma sources covering large areas.

Previous indications of a low-field peak taken with Nagoya type III antennas in uniform magnetic fields are shown in figure 1. Figure 1(a) shows the low-field density peak observed by Chen and Decker [1] in a $2 \mathrm{~cm}$ diameter tube. Figures $1(b)$ and $(c)$ show low-field peaks seen by Chen and Chevalier [2] in $2 \mathrm{~cm}$ diameter and $4 \mathrm{~cm}$ diameter discharges. In the case of figure 1(c), not only was the peak well defined, but the plasma was found to be unusually quiescent and well matched in the neighbourhood of the peak. Figure $1(d)$ shows unpublished data by Aossey and Chen in a separate $2 \mathrm{~cm}$ diameter experiment, showing that the low-field peak occurs only at high RF power. There was no theoretical reason at the time for such a feature, suggesting that perhaps the peak was the result of an experimental artifact such as impurities from the walls. The present results show a general rise in density as $B_{0}$ is lowered but unfortunately cannot be compared directly with the old results or with the theory because of the non-uniform field.

\section{Transition between helicon and ICP discharges}

Including the effect of finite electron mass $m_{\mathrm{e}}$, the wave magnetic field for helicon waves of the form $\boldsymbol{B}(r) \exp [\mathrm{i}(m \theta+k z+\omega t)]$ follows the equation [4,5]

$$
\delta^{*} \nabla \times \nabla \times \boldsymbol{B}-k \nabla \times \boldsymbol{B}+\delta k_{\mathrm{s}}^{2} \boldsymbol{B}=0
$$




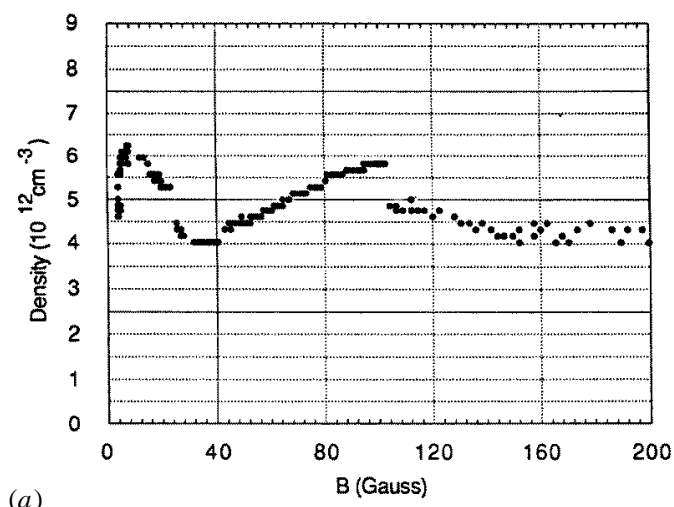

(a)

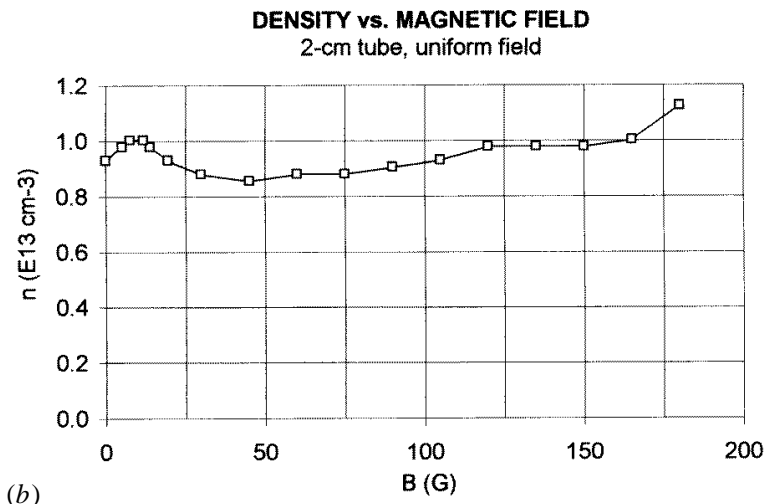

(b)

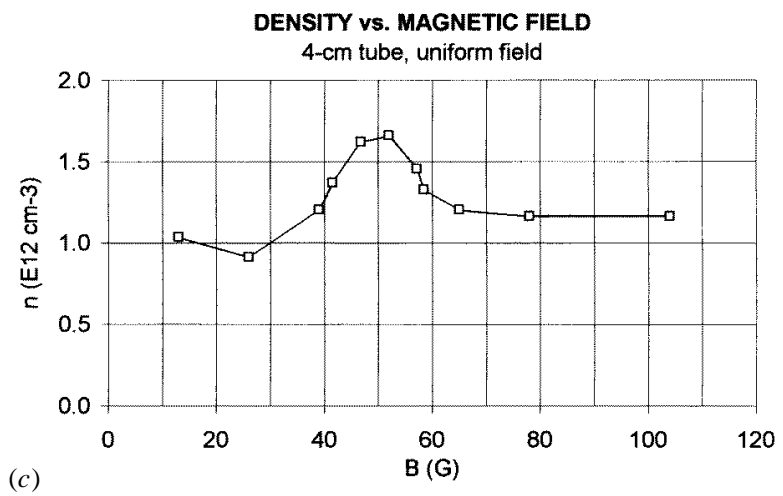

Figure 1. Plasma density on axis against magnetic field, in (a) Chen-Decker experiments [1], in Chen-Chevalier experiments [2] in (b) $2 \mathrm{~cm}$ diameter and (c) $4 \mathrm{~cm}$ diameter tubes, and $(d)$ in Aossey-Chen experiments [3].

where

$$
\begin{aligned}
\delta=\omega / \omega_{\mathrm{c}} \quad \omega_{\mathrm{c}} & =e B_{0} / m_{\mathrm{e}} \quad \delta^{*}=(\omega+\mathrm{i} v) / \omega_{\mathrm{c}} \quad k_{\mathrm{s}}=\omega_{\mathrm{p}} / c \quad \omega_{\mathrm{p}}^{2}=n e^{2} / \epsilon_{0} m_{\mathrm{e}} \\
\delta k_{\mathrm{s}}^{2} & =\omega n_{0} \mu_{0} e / B_{0} .
\end{aligned}
$$

The general solution is $\boldsymbol{B}=\boldsymbol{B}_{1}+\boldsymbol{B}_{2}$, where $\boldsymbol{B}_{1}$ and $\boldsymbol{B}_{2}$ satisfy

$$
\nabla^{2} \boldsymbol{B}_{1}+\beta_{1}^{2} \boldsymbol{B}_{1}=0 \quad \nabla^{2} \boldsymbol{B}_{2}+\beta_{2}^{2} \boldsymbol{B}_{2}=0
$$




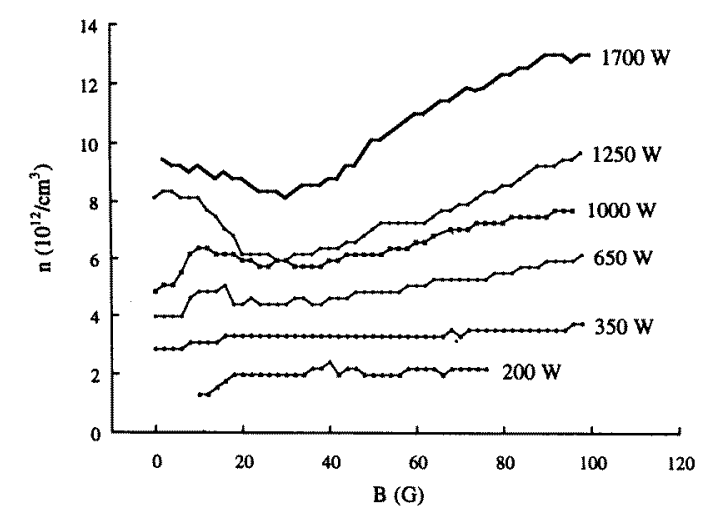

(d)

Figure 1. (Continued)

and $\beta_{1}$ and $\beta_{2}$ are the solutions of

$$
\delta^{*} \beta^{2}-k \beta+\delta k_{\mathrm{s}}^{2}=0
$$

namely

$$
\beta_{1,2}=\frac{k}{2 \delta^{*}}\left[1 \mp\left(1-\frac{4 \delta \delta^{*} k_{\mathrm{s}}^{2}}{k^{2}}\right)^{1 / 2}\right] .
$$

The upper sign gives the helicon $(\mathrm{H})$ branch $\beta_{1}$, and the lower sign the Trivelpiece-Gould (TG) branch $\beta_{2}$. The nature of the normal modes can be seen by neglecting the effective collision frequency $v$ and setting $\delta^{*}=\delta$. Propagating modes then require

$$
k>k_{\min } \equiv 2 \delta k_{\mathrm{s}} \text {. }
$$

For a uniform plasma of radius $a$, solutions of equation (2) can be expressed in terms of Bessel functions $J_{m}(T r)$, where the transverse wavenumber $T$ is given by

$$
T_{j}^{2}=\beta_{j}^{2}-k^{2} \quad j=1,2
$$

Real $T_{j}$ requires $k^{2}<\beta_{j}^{2}$, and equation (3) then gives

$$
k \leqslant k_{\max } \equiv\left(\frac{\delta}{1-\delta}\right)^{1 / 2} k_{\mathrm{s}}
$$

At high magnetic fields, the $\mathrm{H}$ and TG branches are well separated, with $\beta_{2} \gg \beta_{1}$, showing that the TG wave has short radial wavelength. The transition to an ICP discharge occurs in a complicated way. As $B_{0}$ decreases and $\delta$ increases, $k_{\min }$ and $k_{\max }$ approach each other, so that the $\mathrm{H}$ and TG modes, having comparable $\beta$ 's, are strongly coupled. An example of such a solution is shown in figure 2. At a critical field such that $\delta=\omega / \omega_{\mathrm{c}}=1 / 2$, $k_{\min }$ and $k_{\max }$ are equal, and the $\mathrm{H}$ mode disappears. For smaller values of $B_{0}(\approx 17 \mathrm{G}$ at $27.12 \mathrm{MHz}$ ), the $\mathrm{H}$ mode becomes evanescent, with $T_{1}^{2}<0$, and the Bessel functions $J_{m}$ are replaced by $I_{m}$ functions. The TG mode is the only propagating wave, but the coupling to the evanescent $\mathrm{H}$ branch must still be included to satisfy all the boundary conditions. As $B_{0}$ is further reduced toward zero, equation (3) can be written for large $\delta$ as:

$$
\beta_{1,2}=\mp \mathrm{i} k_{\mathrm{s}}\left[\left(1-\frac{k^{2}}{4 \delta^{2} k_{\mathrm{s}}^{2}}\right)^{1 / 2}\right]+\frac{k}{2 \delta} .
$$


Ignoring the small propagating part representing the remnants of the TG mode, we find from equation (6) that $T$ is given by

$$
T \approx \mathrm{i} k_{\mathrm{s}}\left[1-\left(k^{2} / 2 \delta^{2} k_{\mathrm{s}}^{2}\right)^{1 / 2}\right] \equiv \mathrm{i} T^{\prime} .
$$

Here we have taken the positive square root, since there is no energy source for spatial growth in the $-r$ direction. The helicon solutions $J_{m}\left(\mathrm{i} T^{\prime} r\right)$ then become $\mathrm{i}^{m} I_{m}\left(T^{\prime} r\right)$. For $z \gg 1$, the functions $I_{m}(z)$ vary as $\mathrm{e}^{z} /(2 \pi z)^{1 / 2}$. Thus, for $1 / k_{\mathrm{s}} \ll a$, the wave fields decay exponentially as

$$
I_{m}\left(T^{\prime} r\right) \approx \exp \left[k_{\mathrm{s}} r\left(1-k^{2} / 2 \delta^{2} k_{\mathrm{s}}^{2}\right)^{1 / 2}\right] .
$$

The first term in the square root is the usual skin effect in an inductively coupled plasma; the second term gives the increase in penetration because of the magnetization of the electrons, preventing them from short-circuiting the transverse electric field. Such a normal mode analysis does not show why a low-field density peak should exist. Full calculations including antenna geometry and damping are in process, but these can be done only on a case-by-case basis.

\section{Single-tube experiment}

A diagram of the apparatus appears in figure 3. The plasma is created in a $5 \mathrm{~cm}$ diameter quartz tube with an external $m=1$ helical antenna. A magnetic field coil $13 \mathrm{~cm}$ long provides local fields of 0 to $100 \mathrm{G}$. The RF power varied from 400 to $1800 \mathrm{~W}$ at either 13.56 or $27.12 \mathrm{MHz}$. An RF shield (not shown) surrounds the source. The gas fill is typically 18 mTorr of argon. The plasma is injected into a field-free chamber. Probe 1 is a fully RF-compensated Langmuir probe [6] located $6 \mathrm{~cm}$ below the bottom of the source tube. Rapid radial scans can be made with a FastProbe ${ }^{\circledR}$ drive. Probe 2 can be used to measure densities $0.5 \mathrm{~cm}$ below the source.

Figure 4 shows typical radial density profiles from probe 1 when a helical antenna with $m=+1$ symmetry is used. The profiles with different frequencies and magnetic fields are similar. Figure 5 shows the variation of peak density with $B_{0}$ at two frequencies. It is seen that the density decreases with $B_{0}$, and that higher densities are achieved at $27.12 \mathrm{MHz}$ than at $13.56 \mathrm{MHz}$. The antenna length was designed to produce phase velocities that match the velocities of ionizing electrons better at the higher frequency. The density is highest with zero field, and only a hint of the low-field peak is seen. In figure 6 one sees that the peak density generally falls with increasing pressure. Data from probes 1 and 2 are given in figure 7, showing the rapid fall-off of density with distance below the source. Figures 5-7 give no indication of wave generation and suggest that the plasma is created non-resonantly, with the density downstream controlled by diffusion and the divergence of the magnetic field. To better generate helicon-TG waves, the lengths of the tube and of the magnetic field coil were increased by a factor of two with the antenna placed at the upper end. The $n-B$ curves are compared in figure 8 and show that the fall-off with $B_{0}$ is reduced with the long tube, perhaps indicating the influence of wave generation at larger $B_{0}$.

Tests with different antenna designs have also been carried out. An example is given in figure 9, showing $n-B$ curves taken with an $m=0$ antenna consisting of two rings $10 \mathrm{~cm}$ apart carrying current in opposite directions. Here a low-field peak is clearly seen. 


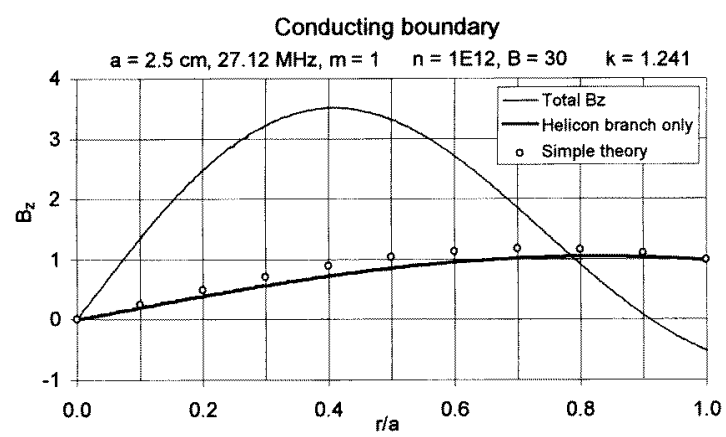

Figure 2. Example of computed $B_{z}$ profile for low magnetic fields ([5]). The thin curve is the total field including the TG branch. The full curve is the field of the helicon branch alone, and the points are computed from zero-electron-mass theory.

\section{SINGLE-TUBE LOW-FIELD EXPERIMENT}

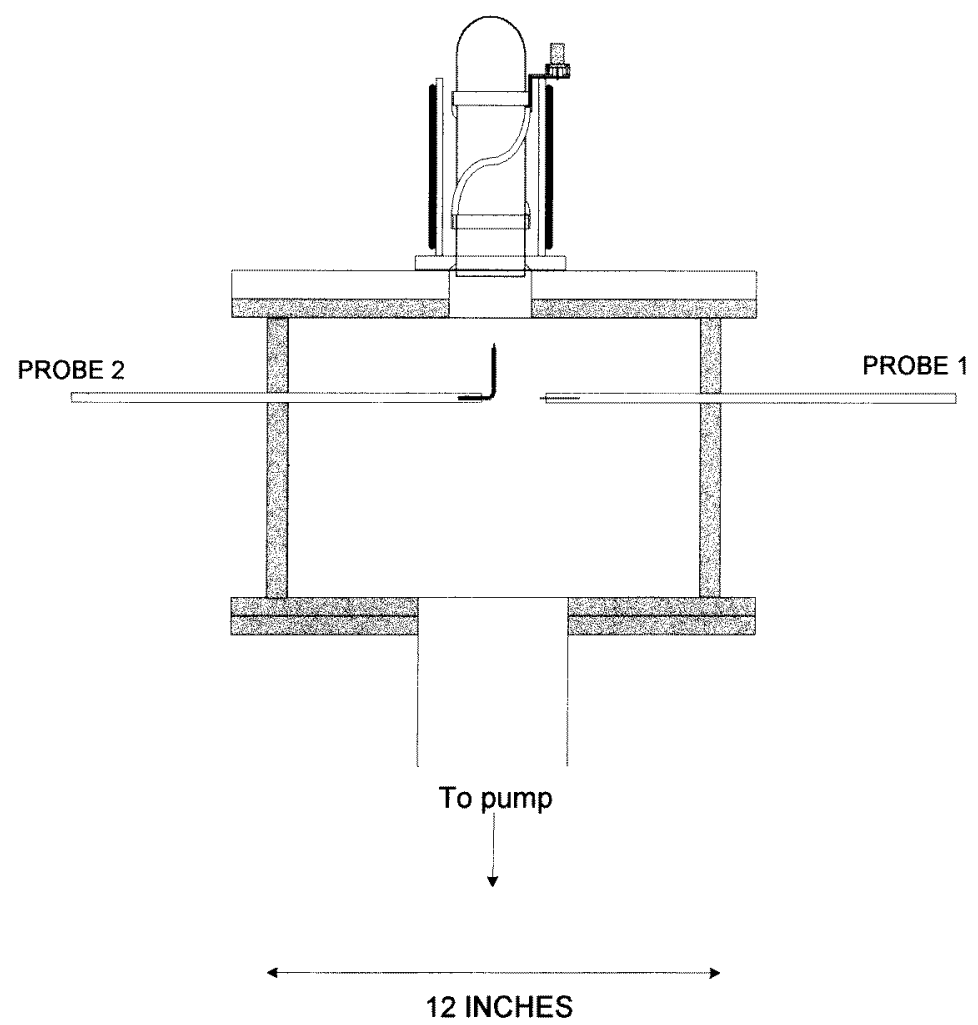

Figure 3. Arrangement of the single-tube experiment.

\section{Multi-tube experiment}

Figure 10 is a schematic of a large-area source (not to scale) consisting of an array of seven tubes, each identical to the one shown in figure 3. The tubes are not in line, as shown, 


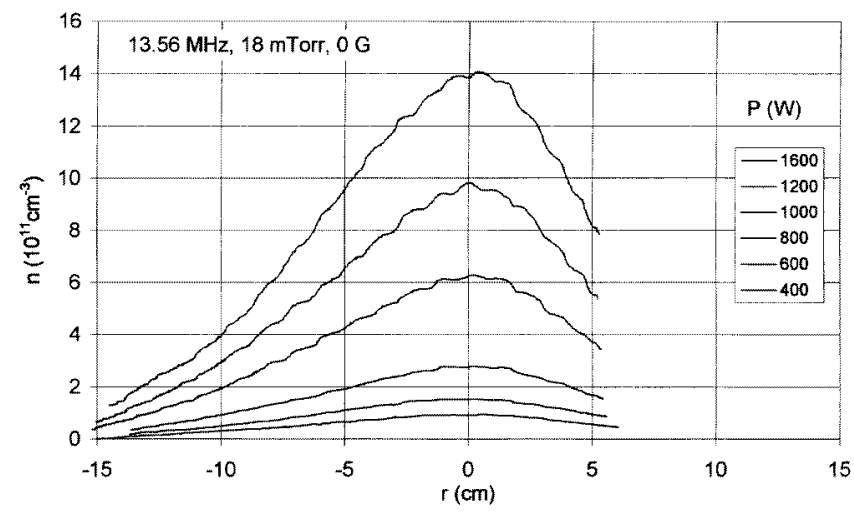

Figure 4. Radial density profiles from the single-tube source at various RF power levels for 13.56 $\mathrm{MHz}$ and $0 \mathrm{G}$. The curves are in the same order as the key.

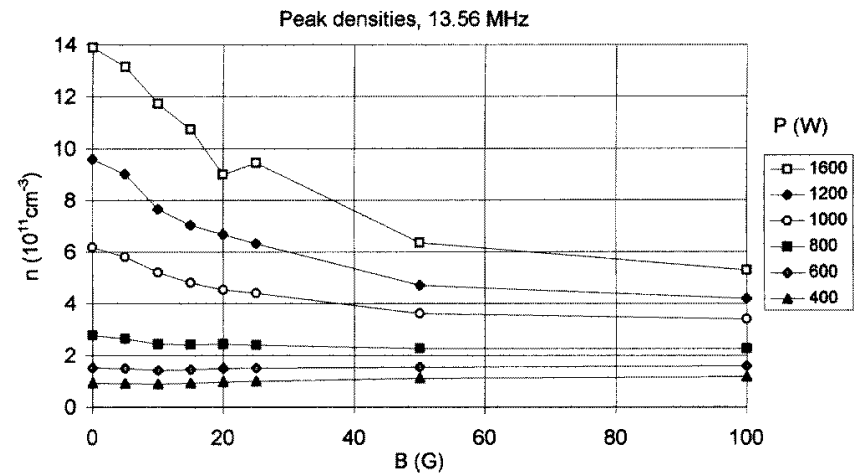

(a)

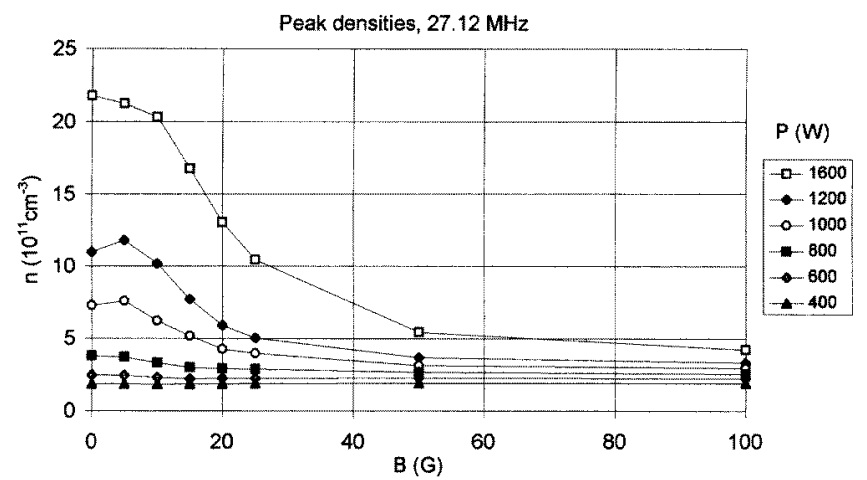

(b)

Figure 5. Peak density against magnetic field at various RF powers for (a) $13.56 \mathrm{MHz}$ and (b) 27.12 Mhz.

but arranged with six tubes on a $20.3 \mathrm{~cm}$ diameter circle and one at the centre. The RF is coupled from a single power supply ( $3 \mathrm{~kW}$ at $13.56 \mathrm{MHz}$ or $2 \mathrm{~kW}$ at $27.12 \mathrm{MHz}$ ) to all 


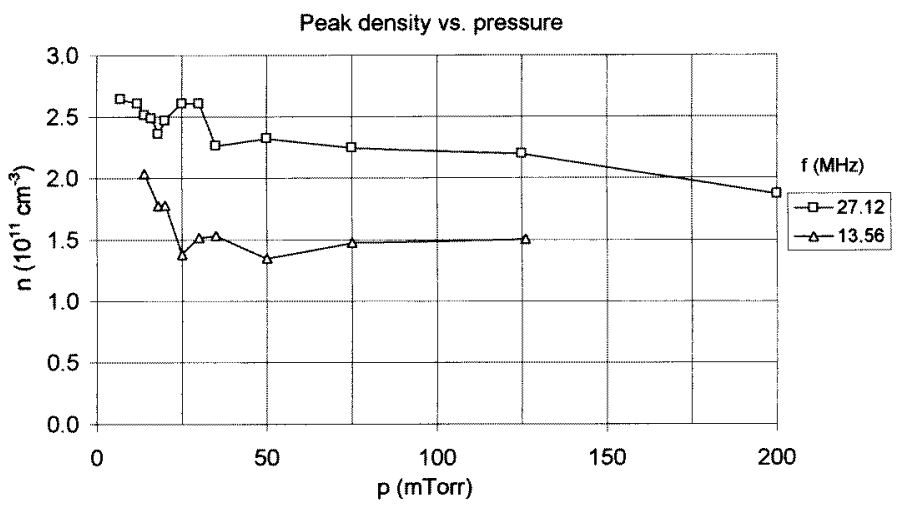

Figure 6. Peak density against argon pressure at $600 \mathrm{~W}$ power and $0 \mathrm{G}$ field, for two frequencies.

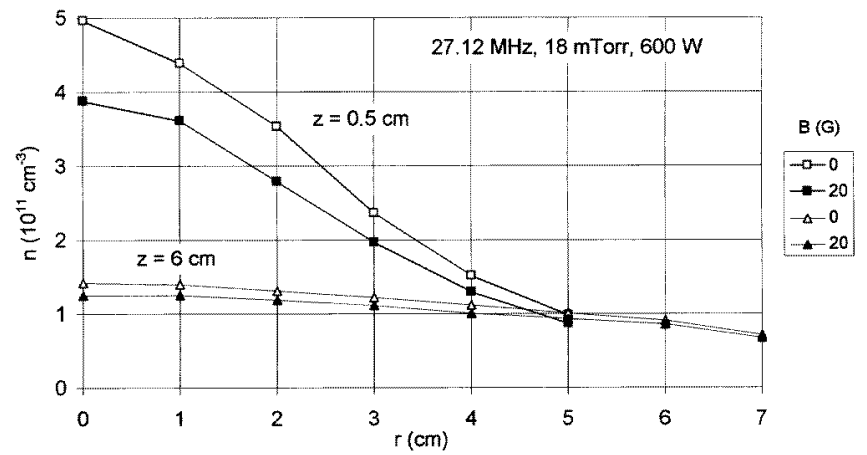

Figure 7. Density profiles at 0.5 and $6 \mathrm{~cm}$ below the source.

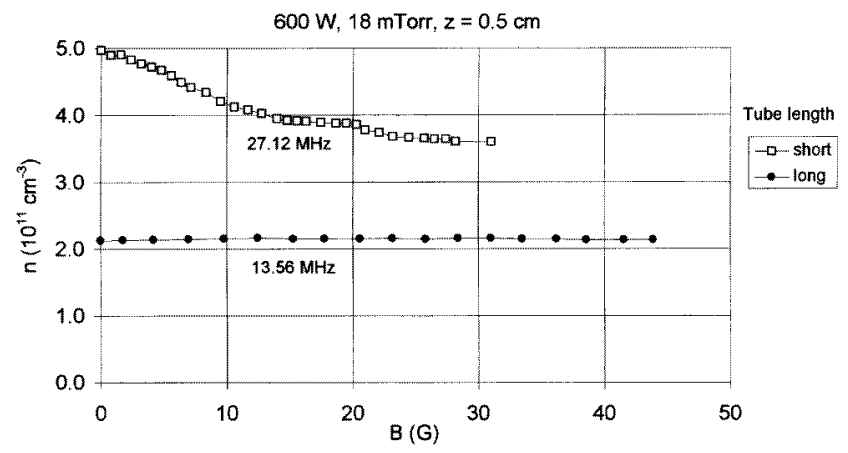

Figure 8. The effect of lengthening the plasma source by a factor of two. (Data at the same frequency were not available.)

seven tubes through a specially designed matching circuit. The plasma is injected into a chamber with multi-dipole surface magnetic confinement. Density distributions are obtained with a Langmuir FastProbe ${ }^{\circledR}$. Optical and magnetic probes are available but have not yet been used. 


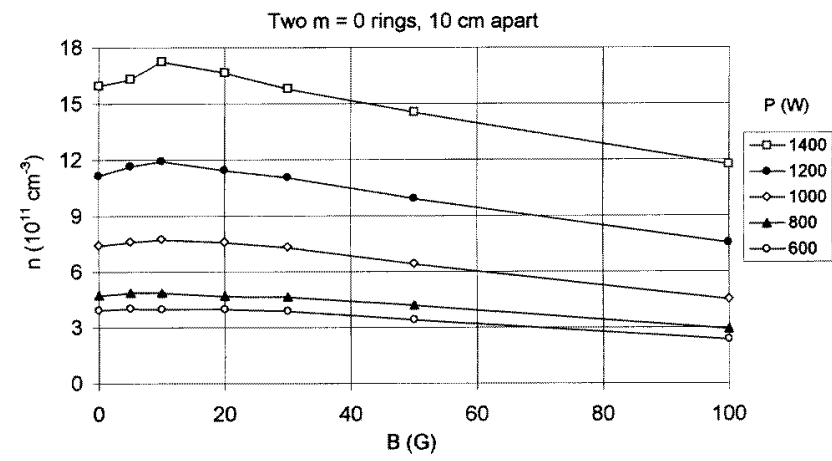

Figure 9. Peak density against magnetic field at $z=6 \mathrm{~cm}$ and 18 mTorr generated by an $m=0$ antenna at various RF powers.

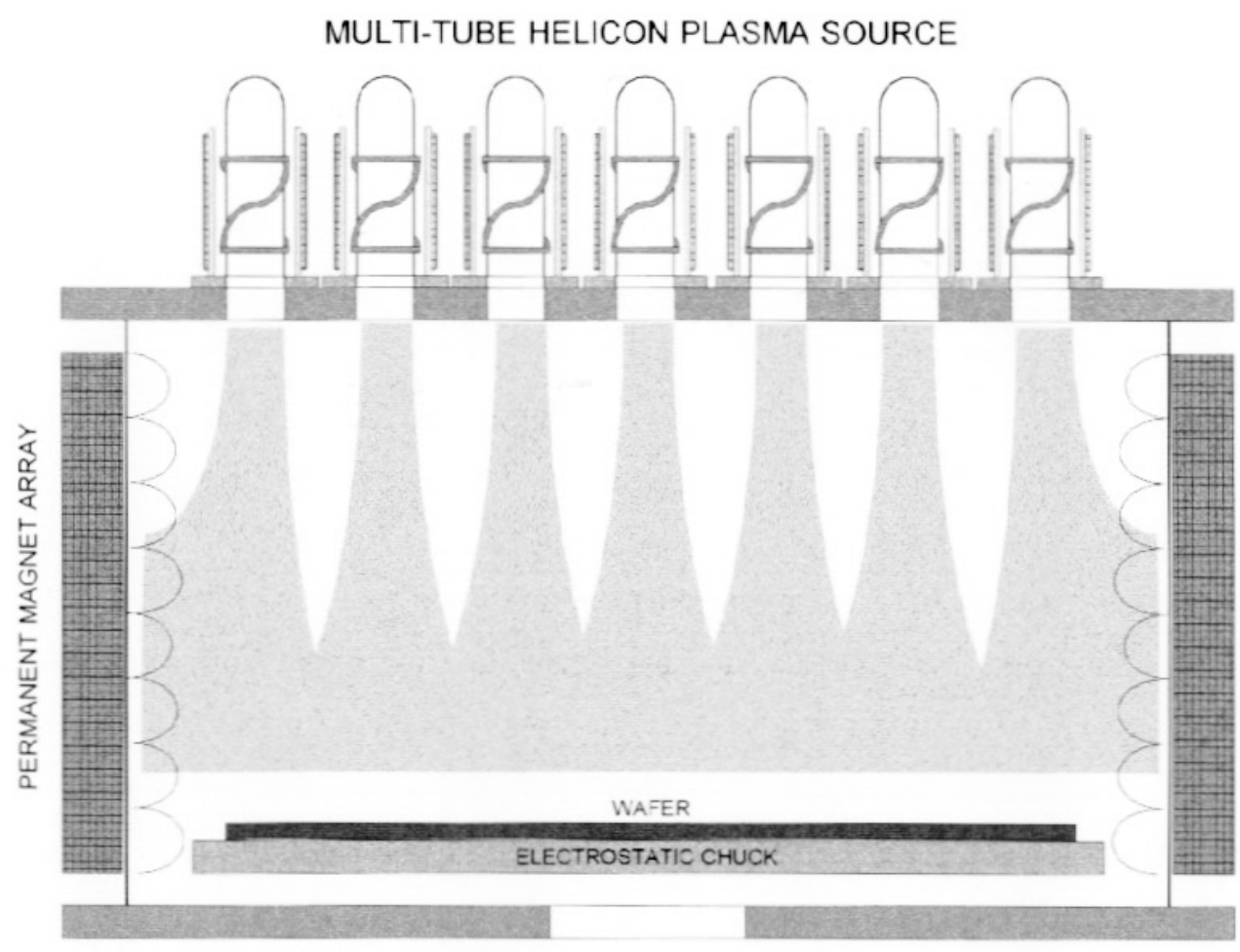

Figure 10. Schematic diagram of a multi-tube, large-area plasma source.

Breakdown is achieved in all seven tubes above about $1 \mathrm{~kW}$ of total power, depending on the gas and pressure. Figure 11 shows radial density distributions $9 \mathrm{~cm}$ below the source in 10 mTorr of $\mathrm{Cl}$ with $3 \mathrm{~kW}$ of $\mathrm{RF}$ power and various values of $B_{0}$. At this distance, the discreteness of the tubes can be seen. The density increases monotonically with $B_{0}$, in contrast with the single-tube case, the difference caused probably by the use of a magnetic bucket. Figure 12 shows density profiles in Ar against $(a) B_{0}$ and $(b)$ RF power. The higher 


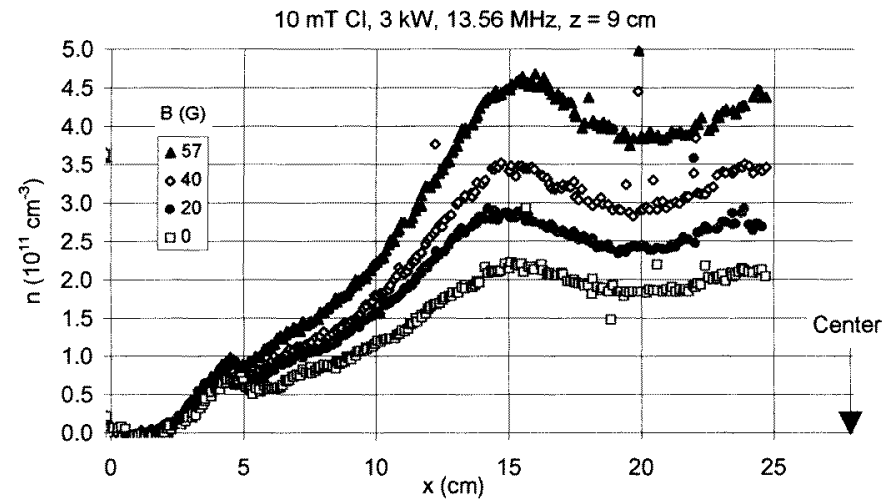

Figure 11. Radial density profiles in the multi-tube source in $\mathrm{Cl}$ gas at various magnetic fields. The probe passes through the axis of one of the circumferential tubes.

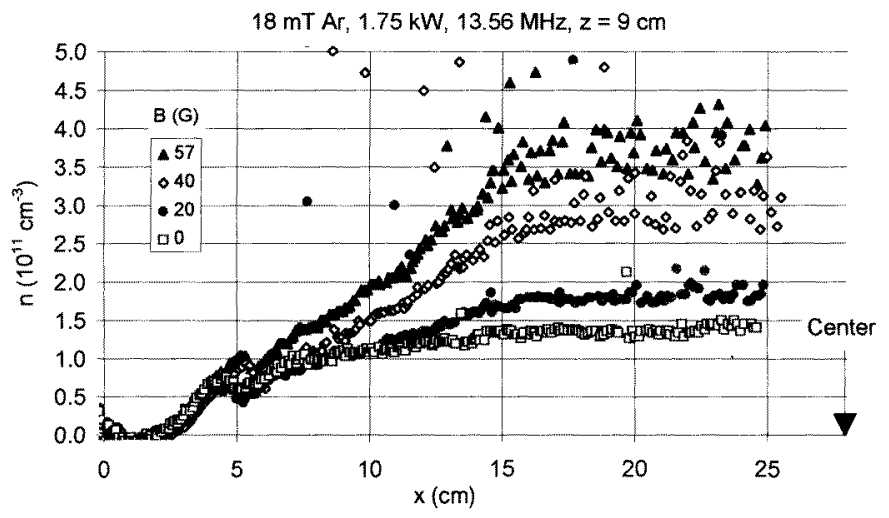

(a)

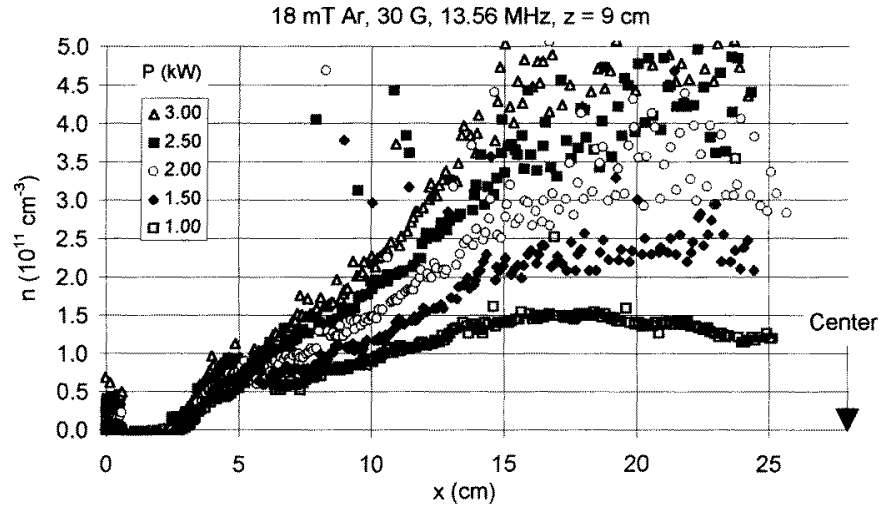

(b)

Figure 12. Radial density profiles in the multi-tube source in $\mathrm{Ar}$ gas at (a) various magnetic fields and $(b)$ various RF powers. 
collisionality in this case has smoothed out the tube structure, even at $z=9 \mathrm{~cm}$. The scatter in the data is caused by RF pickup in the magnet coils. These initial observations will be followed by adjusting the number and placement of the tubes to achieve better uniformity, and ultimately the power in each tube will be set by real-time feedback control.

\section{Conclusions}

Preliminary data with low-field sources show efficient generation of plasma densities in the $10^{12} \mathrm{~cm}^{-3}$ range even with zero magnetic field, but there is so far no indication that the coupled helicon-TG wave plays a role. To compare with theory and to take advantage of the low-field density peak observed previously, further experiments in long, uniform fields are necessary.

\section{Acknowledgments}

This work was supported by the Semiconductor Research Corporation and the National Science Foundation.

\section{References}

[1] Chen F F and Decker C D 1992 Plasma Phys. Control. Fusion 34635

[2] Chen F F and Chevalier G 1992 J. Vac. Sci. Technol. A 101389

[3] Aossey D and Chen F F unpublished

[4] Chen F F 1996 Phys. Plasmas 31783

[5] Chen F F and Arnush D 1996 UCLA PPG-1562, to be submitted

[6] Sudit I D and Chen F F 1994 Plasma Sources Sci. Technol. 3162 